\title{
Axiomatic Multi-Transport Bargaining: A Quantitative Method for Dynamic Transport Selection in Heterogeneous Multi-Transport Wireless Environments
}

\author{
Qiuyi Duan \\ qiuyiduan@gmail.com \\ Michael A. Goodrich \\ mike@cs.byu.edu \\ Charles D. Knutson \\ knutson@cs.byu.edu \\ Lei Wang \\ Follow this and additional works at: https://scholarsarchive.byu.edu/facpub \\ Part of the Computer Sciences Commons
}

\section{Original Publication Citation}

Qiuyi Duan, Lei Wang, Charles D. Knutson, and Michael A. Goodrich. "Axiomatic Multi-Transport Bargaining: A Quantitative Method for Dynamic Transport Selection in Heterogeneous MultiTransport Wireless Environments." Proceedings of the IEEE Wireless Communication and Networking Conference (WCNC 26), Las Vegas, Nevada, April 3-6, 26.

\section{BYU ScholarsArchive Citation}

Duan, Qiuyi; Goodrich, Michael A.; Knutson, Charles D.; and Wang, Lei, "Axiomatic Multi-Transport Bargaining: A Quantitative Method for Dynamic Transport Selection in Heterogeneous Multi-Transport Wireless Environments" (2006). Faculty Publications. 317.

https://scholarsarchive.byu.edu/facpub/317

This Peer-Reviewed Article is brought to you for free and open access by BYU ScholarsArchive. It has been accepted for inclusion in Faculty Publications by an authorized administrator of BYU ScholarsArchive. For more information, please contact ellen_amatangelo@byu.edu. 


\title{
Axiomatic Multi-Transport Bargaining: A Quantitative Method for Dynamic Transport Selection in Heterogeneous Multi-Transport Wireless Environments
}

\author{
Qiuyi Duan, Lei Wang, Charles D. Knutson, Michael A. Goodrich \\ Computer Science Dept. \\ Brigham Young University \\ Provo, UT 84602 \\ \{qiuyi,lei,knutson,mike\}@ cs.byu.edu
}

\begin{abstract}
Transport selection mechanisms are designed to facilitate seamless connectivity in heterogeneous multi-transport environments, allowing access to the "best" available transport according to user requirements. Evaluating transport configurations dynamically according to the user's preferences and Quality of Service $(\mathrm{QoS})$ requirements is a challenging task. This paper describes a quantitative approach that applies the Utility Theorem and Nash's Bargaining solution to heterogeneous wireless environments. The mathematical model presented generates and adjusts the transport preference list dynamically depending on the degree to which a transport satisfies user preferences and the application's QoS requirements. We incorporate a negotiation engine using the Axiomatic Multi-Transport Bargaining algorithm to integrate local and remote users' requirements in a mutually beneficial manner as devices are connected via a peerto-peer link. The transport selection model discussed in this paper is computationally light with modest communication overhead, making it suitable for mobile devices.
\end{abstract}

\section{INTRODUCTION}

Heterogeneous multi-transport devices are increasingly common. Devices in these environments typically possess multiple physical transceivers, such as IrDA, Bluetooth, IEEE $802.11 \mathrm{~b} / \mathrm{g}$, and cellular. Such intra-device heterogeneity can be exploited to optimize connection quality by selecting the "best" available transport, according to the users' preferences and the application's Quality of Service (QoS) requirements.

Quality of Transport (QoT) is a protocol that manages session/application layer access to multiple transports $^{1}$ in heterogeneous wireless environments (Fig. 1). QoT functions as an intelligent layer inserted between the session and transport layers, facilitating dynamic, transparent and autonomous transport switching for multi-transport devices in order to provide the highest quality data transfer capability [5]. If the active transport is disrupted, QoT attempts to connect over an available but less desirable transport without disturbing the session (referred to as a downgrade). If QoT detects that a more preferred transport has become available, it attempts to connect over that transport in order to improve the link quality (referred to as an upgrade). In either case, the selected transport should be the optimal one among available transports, so

\footnotetext{
${ }^{1}$ By "transport" we refer broadly to traditional stovepipe communication architectures interfaced primarily via the transport layer of the protocol stack. Hence, when we use the term "transport," we refer to all layers from the transport layer to the physical layer inclusive. As an example, we would refer to IrDA, Bluetooth and WiFi as separate transports. It should be noted that when we refer to specific protocol stack layers, we specifically include the term "layer" (e.g., "transport layer," "session layer").
}

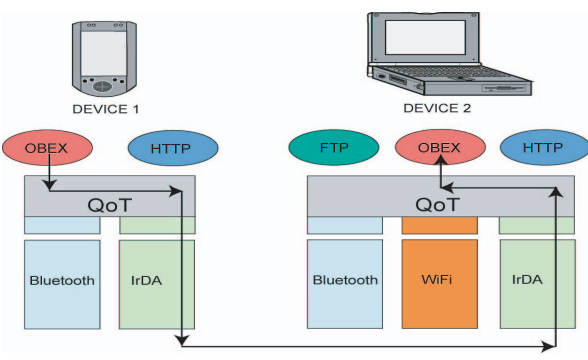

Fig. 1. Data Exchange with QoT in Multi-Transport Environment

that the user's requirements can be maximally satisfied during the communication with QoS commitment.

The transport selection module in the QoT framework is designed to provide and dynamically maintain a transport preference list according to users' preferences and applications' QoS requirements. As a reference for QoT transport switching, such transport preference information allows traffic from session layers to be routed over the "best" available transport at any given time.

The rest of the paper is organized as follows. First, we introduce related work in Section II. Second, we present a transport selection overview in Section III, illustrating the role and functionality of our transport selection model. Next, descriptive criteria selection and user interface are discussed in Section IV and Section V respectively. In Section VI, the transport selection model is introduced in detail. Dynamic preference adjustment is described in Section VII. Finally, conclusions are presented in Section VIII.

\section{RELATED WORK}

A number of research projects have examined heterogeneous connection capabilities. The BARWAN project at UC Berkeley explored the use of vertical handoffs in wireless overlay networks as a mechanism for intelligently and dynamically maintaining an active TCP/IP connection to a network infrastructure [13]. The model assumed that networks with the smallest coverage provided the highest throughput, and hence were the "best." Such network selection may be inappropriate without considering user preferences and other relevant criteria.

The network selection mechanism for the BARWAN project was improved in [14], in which the author proposed a policybased decision making scheme that relied on user input 
to determine tradeoffs between network cost, performance, and power consumption. However, no performance feedback information was provided, and the user was not enabled to adjust requirements after the initial setting. This policy-based approach was further improved in [17] by considering multiple active services, but the feedback problem still exists.

A segment selection algorithm based on fuzzy multiple objective decision making is discussed in [2]. Their model considers the trade-off between cost and quality in order to make a choice between terrestrial and satellite networks for a connection.

The MosquitoNet [1] project at Stanford University was aimed at providing continuous Internet connectivity to mobile hosts through a mechanism of switching seamlessly between different network devices to take advantage of available connectivity, whether wired or wireless. Their work concentrates on Internet connectivity optimization, presuming that Internet access is the only essential usage model. The mechanism by which a connection is chosen is not discussed. The quality of connection and user's preferences are not considered in this model.

A Prioritized Soft Constraint Satisfaction (PSCS) scheme is proposed in [4] to select the "best" transport in a dynamic wireless transport switching system based on a userestablished range of preferences and priority for criteria such as speed, power, range and cost. QoS requirements and mobility issues are not considered in this model. The user interface in this model is relatively complicated, requiring significant user involvement, and the final transport selection is decided almost entirely based on the user's inputs. Non-technical users of the PCSC interface may not understand the meaning of specific terms, or may lack the ability to intelligently specify such criteria. Such weakness may significantly degrade the applicability of the model in practice.

The PSCS transport selection model is extended in [3] for situations in which devices connect over a peer-to-peer link. A negotiation engine is added to generate a preference list that is favorable to both users. However, QoS, mobility, and the complexity and dependency problems are inherited, making the model somewhat weak with respect to accuracy and applicability.

While leveraging some of the strengths of PSCS, the model discussed in this paper generates and adjusts the transport selection list based on the application's QoS requirement and the user's preferences. Performance feedback information is provided for the user's reference and for dynamic adjustment. This model is computationally light with modest communication overhead, and is less dependent on the user's inputs. Besides the intra-device transport evaluation and inter-device preference negotiation scenarios presented in this paper, the proposed model may be applicable to other multi-criteria selection problems in ad-hoc networks.

\section{Transport Selection OVERVIEW}

In order to better understand our model, we present an overview in this section, showing the performing environment, functional role and specific functionality of our transport selection model.

\section{A. Mobility Issue}

Connectivity quality (such as packet loss, error rate, latency, and jitter) changes as a user moves. For example, the signal quality degrades as a user leaves the service range. Hence, user mobility may impact transport selection.

However, computation and communication complexity increase significantly when mobility is considered. Transport selection models require periodic assessment of signal quality for all potential transports, so that the preference list may be updated based on this information. Devices connected over a peer-to-peer link must renegotiate their preference information constantly. Therefore, for complexity and overhead considerations, we separate the measurement of such highly dynamic factors into an independent module.

\section{B. Environmental Role of Transport Selection}

The QoT Brain serves as a "trusted advisor" in the QoT framework. It makes intelligent transport switching decisions autonomously, so that the "best" available transport may be selected at any given time.

As illustrated in Fig. 2, the QoT Brain is composed of submodules. The Transport Selection module is designed to produce and dynamically maintain a preference list, disclosing the desirability of each transport according to user preferences and the application's QoS requirement. The transport preference list is updated whenever a change of preference settings is detected.

The Transport Availability module is used to provide accurate status information for all supported transports via periodic transport performance measurements and quality assessments, in order to minimize the probability of incorrect transport switching. This module considers the QoS parameters that are not involved in the Transport Selection module, such as packet loss, latency, and jitter. Further, it cooperates with the Transport Selection module, providing requisite information for intelligent transport switching decision making.

Query Interval Selection is designed to reduce system overhead by determining an efficient assessment query interval based on the transport desirability and its status records.

The Transport Switching Decision Making module integrates both transport desirability and status information, selects the "best" "stably available" transport at that given moment, and makes the final switching decision.

These four modules are interrelated and function in a cooperative manner. The system shown in Fig. 2 makes it possible to keep the user connected seamlessly over the "best" available transport at any given time. The transport selection model discussed in this paper achieves the functionality of the Transport Selection module in Fig. 2.

\section{DESCRIPTIVE CRITERIA}

QoT-enabled devices rank and select transports transparently without the user's explicit involvement. The user may express specific preferences via a set of descriptive criteria provided by the system, and may make adjustments where desired. Our transport selection model collects such preference settings and translates them into a transport preference list. 


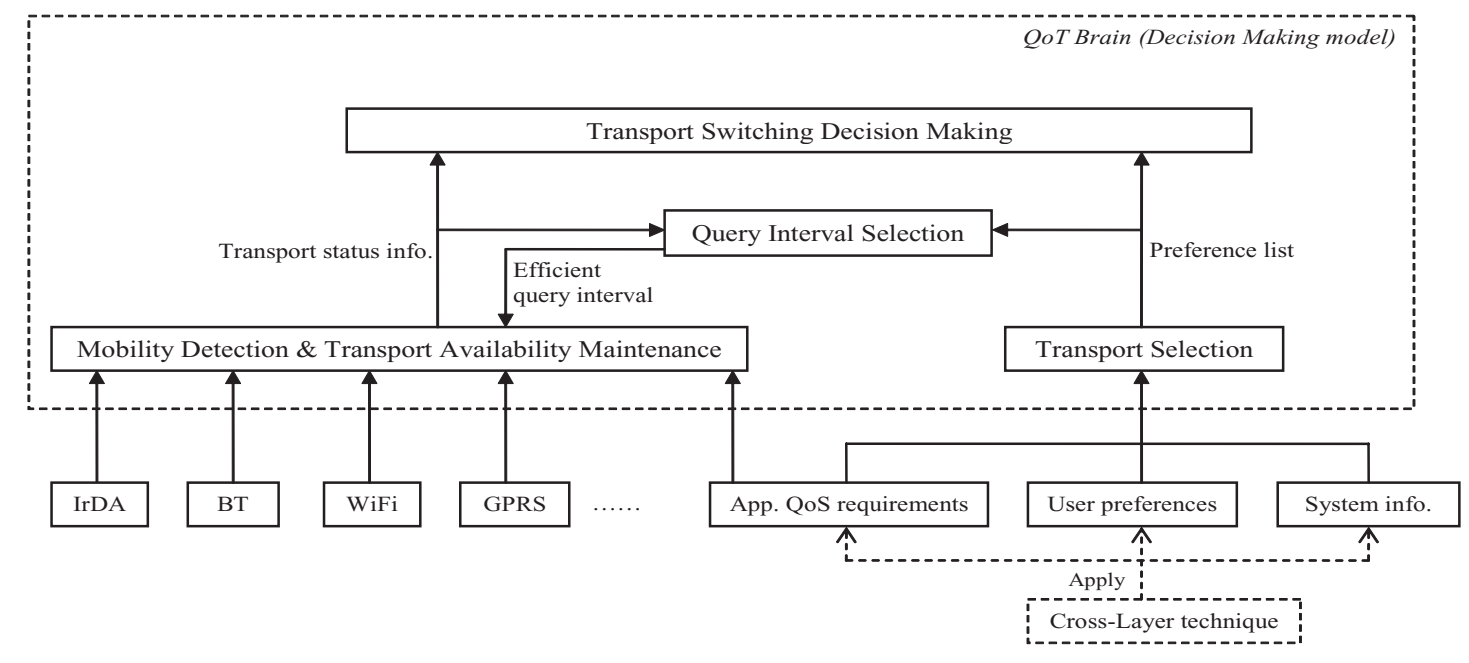

Fig. 2. QoT Brain - Transport Switching Decision Model

Typical descriptive criteria include data rate, power consumption, signal range, service charge, signal quality (latency and reliability), jitter, etc. [4] [2] [14]. We consider two criteria to be critical in our transport selection model: data rate and power consumption.

- Data rate - User preferences may vary with respect to this criterion. Some usage models call for extremely high speed, while others may be satisfied as long as the throughput is sufficient to satisfy the application. The user's preference setting for data rate would be considered as one of transport selection rules during decision making.

- Power consumption - System power is a critical resource for mobile devices. Most users are concerned with the battery life of their mobile devices and some prefer power-efficient services with less data efficiency in order to achieve longer battery duration. Therefore, power consumption is selected as an imperative criterion for transport selection. Users may set specific preferences on this criterion to achieve desirable services.

This criteria set is simple and straightforward, while providing necessary preference information for decision making. Other criteria that we considered but did not include in this model are:

- Service charge - Most short-range wireless services do not require usage fees (for example, IrDA and Bluetooth). Wireless LANs are typically free of charge [14], although the proliferation of subscriber-based WiFi hot spots is changing that somewhat. Still, such hot spots generally operate on a flat-fee basis, rather than a per-byte usage charge. Assuming that a regular flat fee is charged for a user subscription, there is no particular cost savings in avoiding the service. Hence, our current model chooses to ignore service charge as a selection criterion.

- Service range - It is not necessary to consider signal range for mobile communications. For a certain wireless service, the device is either within the signal range or not. Such transport availability variations are considered under the mobility criterion through dynamic transport quality trackings. Furthermore, when the device is under the coverage of a preferred service according to the data rate and power consumption settings, a user's specific restrictions on signal range may even cause incorrect transport selections. Suppose that the user sets the preferred threshold at more than 1 meter, implying that IrDA is not a desirable candidate. If two devices communicate within 1 meter, and both users care a great deal about power consumption. IrDA is the optimal choice. However, it would not be selected due to the range restriction.

- Mobility - Signal quality ${ }^{2}$ changes as a user moves. As discussed in section III, even though mobility is an issue that may impact transport selection, due to its computation and communication overhead, functionality for mobility detection and transport availability maintenance are combined in a separate module.

\section{USER INTERFACE}

The transport selection user interface supports two phases: connection mode selection and preference adjustment.

The interface for connection mode selection, represented in Fig. 3, allows the user to indicate the most important criterion. Selection of high-speed mode indicates that the user is generally more concerned with throughput than with power consumption. Hence, services with high data rate are preferred. The transport with the highest data rate is initially prioritized above other transports. Selection of power-efficient mode demonstrates that the user is more concerned with the device's battery life than with communication speed. Transports with low power consumption are desirable so long as such transports meet the application's throughput requirement. Within all options that are qualified on throughput, the transport with the lowest power consumption is initially prioritized.

\footnotetext{
${ }^{2}$ Signal quality, including packet loss, latency, and jitter etc., is not considered as a selection criterion, since it is difficult for the user to provide specific settings. QoS requirements on these metrics are considered in the mobility module.
} 


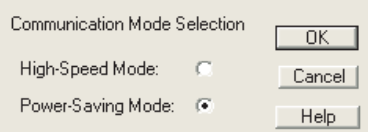

Fig. 3. User Interface: Connection Mode Selection

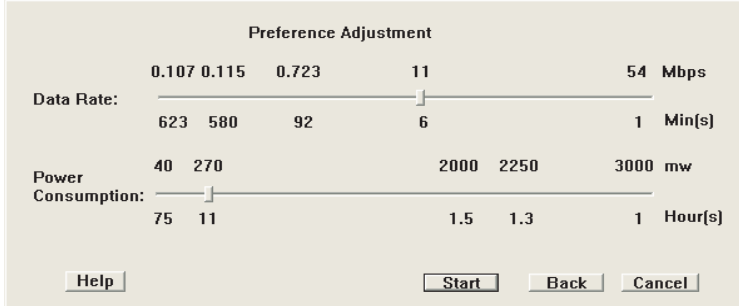

Fig. 4. User Interface: Preference Adjustment

Clearly, connection mode selection is not enough. Sometimes, even though a user chooses high-speed mode, the fastest transport may not be the one that is actually preferred. Thus, mode selection suggests only that the concern for throughput is greater than the concern for power consumption. We need to find a more favorable setting based on the user's specific desires on two criteria. Such information is obtained via the preference adjustment interface, which is illustrated in Fig. 4.

Based on the user's connection mode selection at phase one, two sliders are set to the corresponding positions according to the performance features of the prioritized transport, with data rate and power consumption marked below the axes. Other achievable performance options for data rate and power consumption are distributed along the two axes respectively. If the current setting is satisfactory according to the user's desire (high speed or power saving preference), communication can start immediately. Otherwise, the user can further adjust the setting by moving the slider of the corresponding criterion to other preferred options. For each criterion, the position of the slider indicates the user's preference setting.

Applications have specific Quality of Service (QoS) requirements. Such QoS information may be acquired via crosslayer communication techniques. For example, the application layer could communicate to other layers the application's QoS needs, including required throughput [9]. In order to guarantee a reasonable communication quality, we set the minimum data rate threshold as the QoS throughput requirement. Transports with data rate lower than the minimum threshold are incapable of completing the application, and thus should not be presented as options. Data rate options corresponding to such transports are locked against selection on the interface. The minimum threshold changes as the application's requirement changes.

Criteria are interrelated. A preference setting on one criterion may potentially restrict the selection on the other criterion. For example, in order to provide a data rate higher than the minimum throughput threshold, power consumption may not be made arbitrarily low. As discussed in [4], we use Shannon's Law to determine the minimum power threshold based on the minimum data rate requirement. Any option below the minimum threshold on the power criterion is incapable of meeting the application throughput requirement, and is locked against selection.

For the user's convenience, data rate is also presented as a time representation, namely the elapsed time required to download a one-hour length movie (typically $500 \mathrm{MB}$ ). The power consumption is also transformed into time format, namely the approximate battery life based upon the execution of the target application over the preferred transport. The system power information could also be gathered using crosslayer techniques such as those introduced in [9] [12]. The remaining battery life in our model has a different definition. The purpose of such a transformation is to provide the user an explicit perception of the power consumption features of all transports, instead of an accurate prediction of exactly how long the battery could last considering all tasks and system consumptions. Estimation methods can be found in [16] [10] [8].

\section{Dynamic Transport Selection Model}

The transport selection mechanism is designed to facilitate seamless connectivity while respecting user preferences. The model discussed in this paper dynamically maintains a transport preference list by balancing users' preferences on data rate and power consumption, so that the "best" available transport will be selected at any given time, according to user requirements.

\section{A. Terminology}

We denote $r_{\min }$ as the minimum data rate requirement, which is set based on the QoS throughput requirement gathered from the application layer. The value of $r_{\min }$ adjusts dynamically as the application's requirement changes.

We let $r_{\max }$ represent the maximum data rate, which is the highest data rate that transports can support. The value of $r_{\max }$ is set based on system information, and does not change unless a new transceiver with higher throughput is added.

The minimum and maximum power consumption thresholds are similarly defined, with $p_{\text {min }}$ representing the minimum power threshold (which is set based on $r_{\text {min }}$ using Shannon's Law) and $p_{\max }$ representing the maximum power threshold, which is the highest power consumption regardless of the availability status of transport, according to the system information.

$r_{\text {pref }}$ is used to represent the data rate that the user prefers, gathered from the user interface (see Section V). Any data rate in $\left[r_{\text {pref }}, r_{\text {max }}\right]$ meets the user's preference for throughput. Any data rate in $\left[r_{\text {min }}, r_{\text {pref }}\right)$ is capable of satisfying the application, even though it is not fully desirable.

Similarly, we obtain the user's preference on power consumption from the interface and denote it as $p_{\text {pref }}$. Power consumption in $\left[p_{\min }, p_{\text {pref }}\right]$ meets user's preference, while power consumption in $\left(p_{\text {pref }}, p_{\text {max }}\right]$ is an acceptable option.

$\mathcal{T}=\left\{T_{1}, T_{2}, \ldots, T_{i}, \ldots, T_{n}\right\}$ denotes the set of all transports. $n$ is the number of transports that the device possesses. $T_{i}$ represents transport $i$. 


\section{B. Intra-Device Transport Selection}

The Axiomatic Multi-Transport Bargaining algorithm comprises two phases: intra-device transport preference evaluation and inter-device transport preference negotiation. We first consider phase one ${ }^{3}$, in which the transport is selected according to the local user's preference and the application's QoS requirements.

We apply the Utility Theorem [11][7] to reflect variations in user's requirements. . As the Utility Theorem states, if an agent (user) has a preference relation that satisfies the axioms of preference ${ }^{4}$, then a real-valued utility function ${ }^{5}$ can be constructed that reflects this preference relation such that the following hold:

$$
\begin{gathered}
U\left(T_{i}\right)>U\left(T_{j}\right), \text { if } T_{i} \text { is preferred to } T_{j} \\
U\left(T_{i}\right)=U\left(T_{j}\right), \text { if } T_{i} \text { and } T_{j} \text { are equally preferred }
\end{gathered}
$$

We first set a transport's utility based on the data rate criterion. Transports with data rate less than $r_{\text {min }}$ do not qualify for the requested application. Hence user input is indifferent on those options and 0 is assigned as the data rate utility for all incapable transports. Transports within the range $\left[r_{\text {pref }}, r_{\max }\right]$ are equally desirable according to the user's requirement. We set their data rate utility as 9 . Transports in $\left[r_{\min }, r_{\text {pref }}\right)$ are capable with varying degrees of satisfaction. Their utility is given proportionally in range $[1,9)$ according to their data rate, as shown in Equation 1.

$$
\begin{aligned}
& \frac{r_{T_{i}}-r_{\min }}{r_{\text {pref }}-r_{\text {min }}}=\frac{U_{\text {rate }}\left(T_{i}\right)-U\left(r_{\min }\right)}{U\left(r_{\text {pref }}\right)-U\left(r_{\min }\right)} \\
& \Rightarrow U_{\text {rate }}\left(T_{i}\right)=\frac{r_{T_{i}}-r_{\text {min }}}{r_{\text {pref }}-r_{\min }} * 8+1
\end{aligned}
$$

Therefore, we define the utility function for the data rate criterion as Equation 2 to reflect the user's preferences on available transports. The relationship of utility and data rate is further illustrated in Fig. 5.

$$
U_{\text {rate }}\left(T_{i}\right)= \begin{cases}0 & r_{T_{i}}<r_{\text {min }} \\ \frac{r_{T_{i}}-r_{\text {min }}}{r_{\text {pref }}-r_{\min }} * 8+1 & r_{T_{i}} \in\left[r_{\text {min }}, r_{\text {pref }}\right) \\ 9 & r_{T_{i}} \in\left[r_{\text {pref }}, r_{\text {max }}\right]\end{cases}
$$

Similarly, we create another real-valued function via Equation 3 to represent a user's well-behaved preferences on the power consumption criterion. The utility setting is also illustrated in Fig. 5.

\footnotetext{
${ }^{3}$ The transport selection method at phase one also applies to a scenario in which the mobile device communicates with an infrastructure, such as a network access point.

${ }^{4}$ The axioms of preference refer to the properties of orderability, transitivity, continuity, substitutability, monotonicity, and decomposability. Details can be found in [11].

${ }^{5}$ The utility function is unique up to a positive affine transformation, which means that if a function $U()$ satisfies the axioms of preference for a particular agent, then so does $\alpha U()+\beta$ for $\alpha>0$ and $\beta \in R$.
}
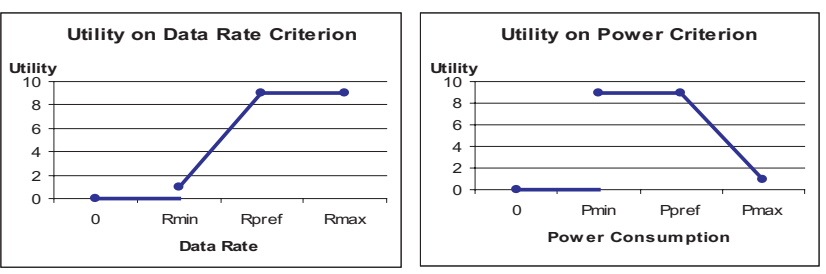

Fig. 5. Relationship of Utility and Descriptive Criteria

$$
U_{\text {power }}\left(T_{i}\right)= \begin{cases}0 & p_{T_{i}}<p_{\text {min }} \\ 9 & p_{T_{i}} \in\left[p_{\min }, p_{\text {pref }}\right] \\ \frac{p_{\max }-p_{T_{i}}}{p_{\max }-p_{\text {pref }}} * 8+1 & p_{T_{i}} \in\left(p_{\text {pref }}, p_{\max }\right]\end{cases}
$$

For simplicity, we use 0 and real numbers from 1 to 9 to represent the utilities. Any real number will do, provided that the number assigned to the most preferred option is higher than the number assigned to the least preferred option and connected devices use the same scale.

Now, according to the user's preferences, each transport is evaluated by two independent criteria, data rate and power consumption, via the Equation 2 and 3 respectively. However, these two criteria are not equally important from the user's standpoint. We need to balance their degrees of importance when calculating the transport's overall utility. We do this by taking a weighted combination of the utilities of data rate and power consumption using a weight that matches the user's connection mode selection, where we can determine the criterion that is more important. In our model, this criterion is weighted 10 times more heavily than the less important criterion. The overall utility for each transport is calculated using Equation 4, where the value of the more important criterion is put to the tens position to represent its stronger influence on the outcome.

$U_{\text {overall }}\left(T_{i}\right)= \begin{cases}U_{\text {rate }}\left(T_{i}\right) * 10+U_{\text {power }}\left(T_{i}\right) & \text { high-speed } \\ U_{\text {power }}\left(T_{i}\right) * 10+U_{\text {rate }}\left(T_{i}\right) & \text { power-efficient }\end{cases}$

It is possible for us to choose other weight values to reflect the importance of the more important criterion. Experiments were conducted to determine the effects of the weight selection in Equation 4. Although not shown because of space limitations, we found that smaller weights were not strong enough to reflect the relative importance of the criterion in the utility, and larger weights neglected the less important criterion too much. Since the utility range for each criterion is $[0,9]$, weights that are greater than 9 are sufficient to disclose the decisive influence of the more important criterion and provide similar balanced information. We empirically selected 10 as the balancing weight in our model.

The transport with higher overall utility ranks higher in the final transport preference list and is more preferred by the user. As implied in Equation 4, the transport with the higher utility on the more important criterion better meets the user's preference and QoS requirements. Hence, it is assigned a higher overall score and is ordered higher for selection. 
Transports with the same utility evaluations on the more important criterion are ordered based on their utilities on the other criterion. The transport with a satisfying performance on the less important criterion may not be desirable - its probability of being selected depends on its performance on the more important criterion. The overall utility of transports that are unqualified on either criterion $\left(U_{\text {rate }}\left(T_{i}\right)=0\right.$ or $U_{\text {power }}\left(T_{i}\right)=0$ ) is set to 0 . Transport with a lower power consumption is preferred if there is a tie.

The overall utility explicitly represents the user's preference with QoS consideration. The transport is selected based on the degree to which it satisfies user requirements.

\section{Inter-Device Transport Selection}

As devices are connected over a peer-to-peer link with differing preferences on connection quality - a transport selection preferred by one user may not satisfy the other user's requirements. A transport preference negotiation mechanism is required to integrate both local and remote users' preferences. In this section, we consider the second phase of Axiomatic Multi-Transport Bargaining algorithm - inter-device preference negotiation.

Traditional turn-taking negotiation incorporates significant communication overhead and inherits potential deadlock risks. In order to solve this problem, QoT employs a third party arbitration handled through the arbitration engine on the primary device [3]. The engine works like a benevolent court system, which can fairly enforce the agreement reached by the agents.

The arbitration engine first gathers preference information from local and remote devices on common transports. Only the transport's overall utility is required, since it includes all relevant information, such as the connection mode selection, the application QoS requirement, and the user's specific preferences on each descriptive criterion. Based on such preference information, we apply Nash's Axiomatic Bargaining Solution [15] [7] [6] to evaluate all transport configurations and generate the commonly shared social preference list.

Nash's Axiomatic Bargaining is a mechanism that sets up the rules of the negotiation so that socially "good" things happen given any users' utilities. A transport that is mutually beneficial to both users is preferred. The better it meets both users' requirements, the higher it is listed.

As Nash's Axiomatic Bargain algorithm defines, there is a special outcome, called a fall-back solution, that can result if negotiation breaks down. As transport selection negotiation fails, no transport can satisfy both users' requirements simultaneously, and no connection can be set up. Therefore, we set the utility of $f b$ solution as $U_{\text {user } 1}(f b)=U_{\text {user } 2}(f b)=0$.

According to Nash's Axiomatic Bargaining algorithm, the best negotiation solution is the one that maximizes the benefits of the two agents, as demonstrated in Equation 5:

$T_{\text {best }}=\underset{T_{i} \in \mathcal{T}}{\arg \max }\left[U_{\text {user } 1}\left(T_{i}\right)-U_{\text {user } 1}(f b)\right]\left[U_{\text {user } 2}\left(T_{i}\right)-U_{\text {user } 2}(f b)\right]$

Since $U_{\text {user } 1}(f b)=U_{\text {user } 2}(f b)=0$, Equation 5 can be simplified into Equation 6.

$$
T_{\text {best }}=\underset{T_{i} \in \mathcal{T}}{\arg \max }\left[U_{\text {user } 1}\left(T_{i}\right)\right]\left[U_{\text {user } 2}\left(T_{i}\right)\right]
$$

Applying Nash's Axiomatic Bargaining algorithm, we define a real-valued function, namely the Axiomatic MultiTransport Bargaining algorithm, to calculate the social utility for each transport using Equation 8 and make a social selection using Equation 7.

$$
\begin{gathered}
T_{\text {best }}=\underset{T_{i} \in \mathcal{T}}{\arg \max } U_{\text {social }}\left(T_{i}\right) \\
U_{\text {social }}\left(T_{i}\right)=U_{\text {user } 1}\left(T_{i}\right) * U_{\text {user } 2}\left(T_{i}\right)
\end{gathered}
$$

Preferences of both local and remote users are equally considered during the negotiation. A transport's social utility is calculated based on its overall utility evaluated by the two connecting devices. The higher the utility a transport gets, the better it meets the users' preferences simultaneously. The transport with the highest utility value is the best negotiation solution as defined by the Axiomatic Bargaining algorithm.

Social selection should be fair with respect to both users' requirements. A fair solution in our model implies two aspects. One is that the negotiation process is unbiased, meaning that the connecting users' preferences are impartially considered. The other is that the selected solution is Pareto optimal, meaning that no alternative solution is better than the selected one for both users. A transport that simultaneously satisfies both users' requirements in a mutually beneficial manner is preferred.

Nash's Axiomatic Bargaining algorithm is fair according to its axioms. It is Pareto optimal, symmetric, independent of the utility scales of two agents, and independent of irrelevant alternatives [6]. The Axiomatic Multi-Transport Bargaining algorithm inherits such features and is able to provide a fair solution that satisfies the above fairness axioms. Our algorithm allows the "best" available transport to be selected via an impartial consideration of the preferences from both sides.

The social transport selection list is ordered from the transport with the highest social utility to the one with the lowest value. A transport with lower power consumption is preferred if there is a tie. If the active transport becomes unavailable, connecting devices could switch to the next available transport, which is currently the "best" transport among all available options. As a more preferred transport becomes available, connecting devices could upgrade to that transport to achieve a more favorable connectivity quality. Therefore, the most preferred available transport configuration could be selected at any given time, providing a mutually beneficial connectivity.

This transport selection model performs with reasonable computation and communication overhead because of its simplicity, making it suitable for mobile devices.

\section{Performance Evaluation}

We employed two QoT-enabled devices in our experiments, connecting via a peer-to-peer link in order to exchange files using the OBEX protocol. The commonly shared transports are IrDA (SIR), Bluetooth, IEEE 802.11b/a, and GPRS. We vary 


\begin{tabular}{|l|c|c|}
\hline Transport & Data Rate(Mbps) & Power (mw) \\
\hline IrDA (SIR) & 0.115 & 40 \\
\hline Bluetooth & 0.723 & 270 \\
\hline IEEE 802.11b & 11 & 2250 \\
\hline IEEE 802.11a & 54 & 2000 \\
\hline GPRS & 0.107 & 3000 \\
\hline
\end{tabular}

Fig. 6. Performance Scenario Data of All Transport Configurations

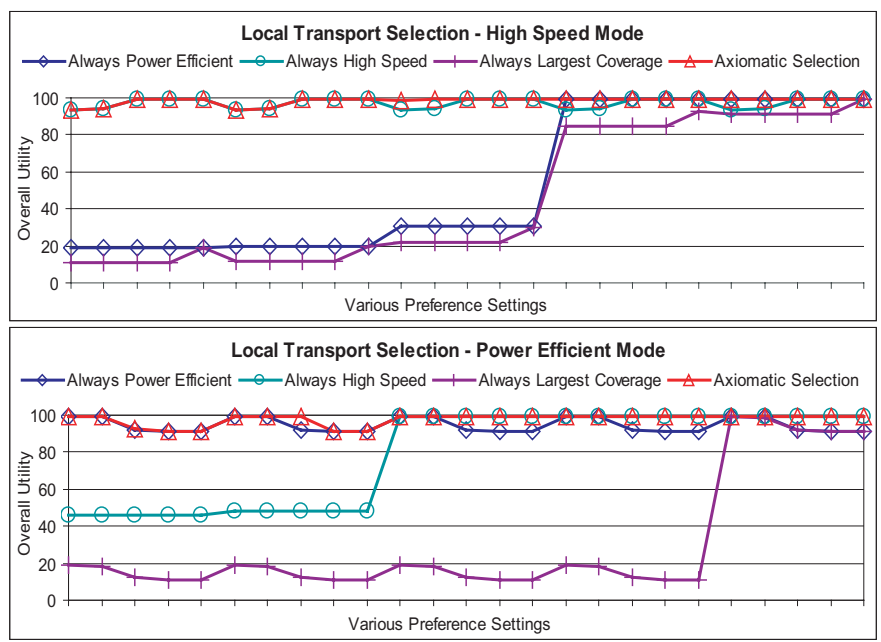

Fig. 7. Intra-Device Transport Selection Comparisons on Various Preference Settings - Desirability of the Selection

users' inputs to all possible mode and preference settings, and compared the performance of our algorithm with three other strategies: Always Power Efficient, Always High Speed, and Always the Largest Coverage. The minimum and maximum thresholds for data rate and power consumption were gathered from the application layer and system information using cross-layer techniques. Scenario data, shown in Fig. 6, were collected from each transport's specification.

We use two metrics to evaluate the performances of various selection algorithms: desirability and fairness.

- The desirability metric reflects the degree to which a selected transport satisfies the users' requirements. We use a transport's overall utility for intra-device selection evaluation, and the average overall utility of the transport over thousands of different preference settings from both users for performance comparison on inter-device selection.

- The fairness metric indicates the degree of impartiality with which users' preferences are considered during negotiation. Like desirability, we use the average overall utility of the transport over all possible preference settings from both users for such performance evaluation.

Fig. 7 compares our algorithm with the other three strategies for local transport selection using the desirability metric ${ }^{6}$. As the result demonstrates, the transport selected using our algorithm is consistently more or equally desirable when compared to other strategies. The "best" available transport

\footnotetext{
${ }^{6}$ The $\mathrm{X}$-axis represents all possible preference settings under a specific connection mode selection. Patterns of the lines in Fig. 7 depend on the experiment order on various settings, and are not essential to the motivations that underlie the research presented here.
}

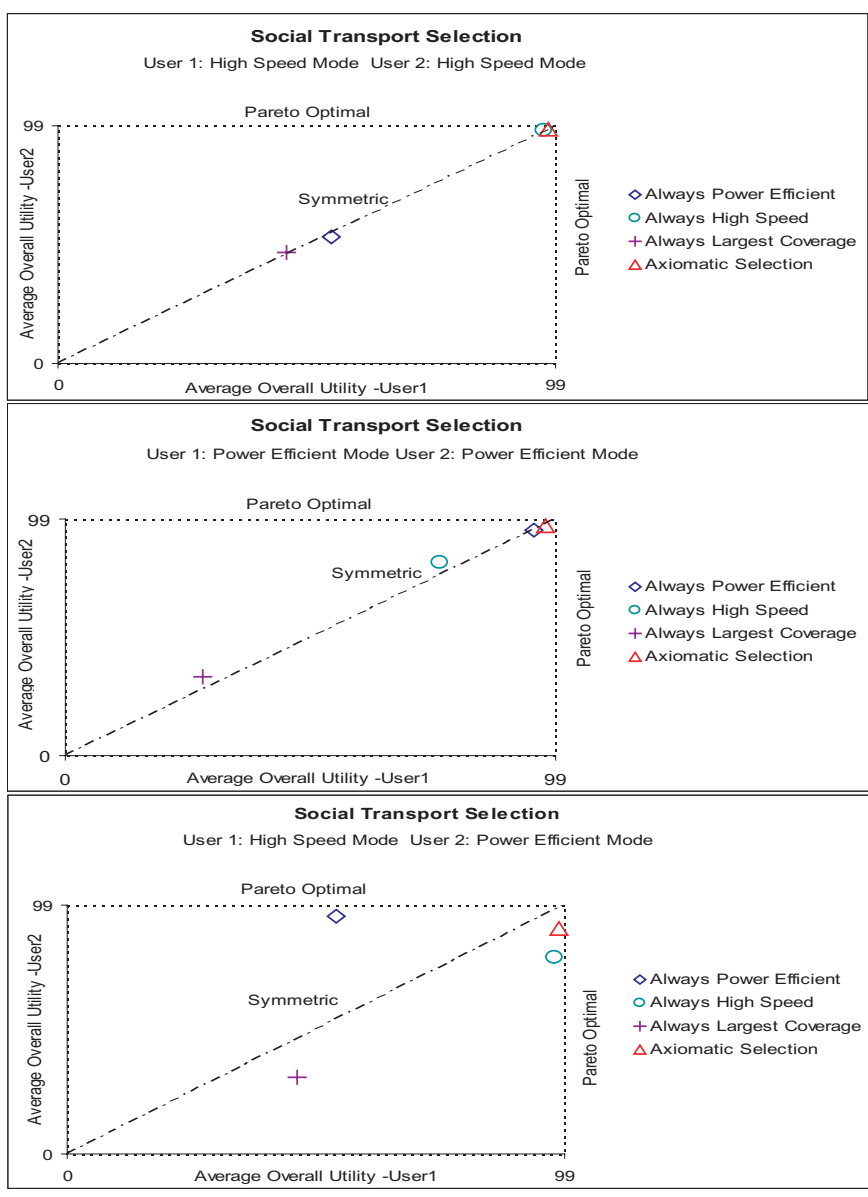

Fig. 8. Inter-Device Transport Selection Comparisons on Various Mode and Preference Settings — Fairness and Desirability of the Selection

could be selected following our transport preference list in either High-Speed or Power-Efficient mode.

Fig. 8 compares the fairness and desirability of our algorithm with the other three strategies in three cases: both users care about speed; both users care about power consumption; users have different connection mode preferences. The result of each case is the average overall utility of the selected transport over all possible preference setting combinations with respect to that specific mode selection.

According to the line of symmetry, Fig. 8 indicates that the transport selected using the Axiomatic Multi-Transport Algorithm is consistently fairer than the selection using the other strategies in all cases. When two users make the same connection mode selections, they have similar evaluations on the commonly shared transports. Hence, transport selection of any strategy meets the connecting users' requirements with a similar degree of satisfaction, either desirable to both sides or undesirable to both sides. As the figure shows, the transport selected using our algorithm is closer to the line of symmetry compared to the selection of other strategies, even though the advantage is not significant. When two users make different connection selections, their preferences may be significantly different. The Axiomatic Multi-Transport algorithm dynamically evaluates all shared transports according to both users' requirements and selects the option that is mutually beneficial 
to both sides. As the figure shows, the transport selected using our algorithm is much closer to the line of symmetry compared to the selection of other strategies.

Based on the Pareto optimal lines, Fig. 8 demonstrates that the transport selection using the Axiomatic MultipleTransport Bargaining algorithm is consistently more desirable when compared to the other three strategies in any case with all different preference setting combinations from the connecting peers. The Always High Speed and Always Power Efficient strategies only perform well when both users concern speed and both concern power consumption respectively. The transport selected using the Axiomatic Multi-Transport algorithm is consistently the most desirable one compared to other strategies, because of its capability of choosing the Pareto optimal option through dynamic evaluations. The advantage is especially obvious when connecting users have different mode selections.

As the experimental results illustrate, our algorithm allows the "best" available transport in terms of fairness and desirability to be chosen, so that both users' connectivity requirements are mutually benefited during the communication.

\section{Dynamic Preference Adjustments}

The preference adjustment interface (Fig. 4) also provides feedback information on connection quality. Positions of the two sliders are dynamically adjusted according to the current transport configuration, disclosing present connection performance information.

The user can adjust the preference setting at any time as requirements change, by moving the corresponding slider to the desired option or by changing the connection mode selection. Relevant variables, such as $r_{\text {pref }}, p_{\text {pref }}$, and connection mode, change accordingly. The transport selection model reevaluates all transport configurations based on the updated information, and generates a preference list according to the new preference setting. A QoT enabled device would then switch to the most desired available transport, so that the configuration selected is always favorable to the user's requirement.

The transport preference list also changes as the application's QoS requirement changes or as the system resources change.

As introduced in section $\mathrm{V}$, minimum thresholds of the two criteria are set based on an application's QoS requirements. As an application executes, QoS requirements change accordingly. With cross-layer information sharing, QoT could detect such changes and adjust $r_{\min }$ and $p_{\min }$ dynamically according to the new requirement.

Maximum thresholds of the two descriptive criteria, $r_{\max }$ and $p_{\max }$, are set based on system resource information. Their values do not changed unless a new transceiver with an even higher data rate or power consumption feature is added.

The transport selection model reevaluates all supported transports based on the newly updated criteria information. The social preference list is also updated through renegotiation as devices are connected over a peer-to-peer link.

\section{CONCLUSION}

This paper presents a dynamic transport selection model that applies the Utility Theorem and the Axiomatic Bargaining algorithm to heterogeneous wireless environments. The mathematical model generates and adjusts a preference list dynamically, facilitating seamless connectivity with user preferred quality. Multiple users' requirements are satisfied in a mutually beneficial manner using Axiomatic Multi-Transport Bargaining algorithm. The model is suitable for mobile devices with modest computation and communication overhead.

Future work in transport selection decision making could be focused on multiple transport utilization, such as inverse multiplexing, making the connectivity more favorable according to user preference.

\section{REFERENCES}

[1] M. Baker, X. Zhao, and J. Stone. Supporting mobility in mosquitonet. In proceedings of the 1996 USENIX Technical Conference, San Diego, California, 1996

[2] P.M.L. Chan, Y.F. Hu, and R.E. Sheriff. Implementation of fuzzy multiple objective decision making algorithm in a heterogeneous mobile environment. In Proceedings of the IEEE Wireless Communications and Networking Conference, Orlando, FL, USA, March, 2002.

[3] Heidi R. Duffin, Charles D. Knutson, and Michael A. Goodrich. Negotiating transport selection between qot-enabled devices. Technical report, Mobile Computing Lab., Brigham Young University, 2003.

[4] Heidi R. Duffin, Charles D. Knutson, and Michael A. Goodrich. Prioritized soft constraint satisfaction: A qualitative method for dynamic transport selection in heterogeneous wireless environments. In Proceedings of the IEEE Wireless Communications and Networking Conference (WCNC), 2004.

[5] Charles D. Knutson, Ryan W. Woodings, Shannon B. Barnes, Heidi R. Duffin, and Jeffrey M. Brown. Quality of transport (qot): Dynamic autonomous transport selection in heterogeneous wireless environments. In Proceedings of the IEEE Wireless Communications and Networking Conference (WCNC), 2004.

[6] Abhinay Muthoo. Bargaining Theory with Applications. Cambridge University Press, 1999.

[7] Jr. J. F. Nash. The bargaining problem. Econometrica, 18:155-162, 1950. Reprinted in Classics in Game Theory, H. W. Kuhn, ed.

[8] D. Panigrahi, C. Chiasserini, S. Dey, R. Rao, A. Raghunathan, and K. Lahiri. Battery life estimation of mobile embedded systems. In The 14th IEEE International Conference on VLSI Design, 2001.

[9] Vijay T. Raisinghani and Sridhar Iyer. Cross layer design optimization in wireless protocol stacks. Submitted to Elsevier Science, Mar. 2, 2003.

[10] D. Rakhmatov, S. Vrudhula, and D. A. Wallach. Battery lifetime prediction for energy-aware computing. In In Proceedings of the International Symposium on Low Power Electronics and Design, Augest 2002.

[11] S. Russell and P. Norvig. Artificial Intelligence: A Modern Approach. Prentice Hall, 1995.

[12] Sanjay Shakkottai, Theodore S. Rappaport, and Peter C. Karlsson. Cross-layer design for wireless networks. In Communications Magazine, IEEE, volume 41, pages 74 - 80, Oct. 2003.

[13] M. Stemm and R. H. Katz. Vertical handoffs in wireless overlay networks. Mobile Networks and Applications, 4, 1999.

[14] H. J. Wang, R. H. Katz, and J. Giese. Policy-enabled handoffs across heterogeneous wireless networks. In Second IEEE Workshop on Mobile Computing Systems and Applications (WMCSA '99). IEEE, 1999.

[15] Gerhard Weiss, editor. Multiagent Systems : A Modern Approach to Distributed Artificial Intelligence. Cambridge, Mass. MIT Press, 1962, C1999.

[16] Ye Wen, Rich Wolski, and Chandra Krintz. Online prediction of battery lifetime for embedded and mobile devices. Technical report, Computer Science Department, University of California, Santa Barbara, June 2003.

[17] Fang Zhu and J. McNair. Optimizations for vertical handoff decision algorithms. In IEEE Wireless Communications and Networking Conference (WCNC), volume 2, pages 867-872, Mar. 2004. 